\title{
Mitochondrial Targeting and pH-Responsive Nanogels for Co-Delivery of Lonidamine and Paclitaxel to Conquer Drug Resistance
}

\author{
Enping Chen ${ }^{1 \dagger}$, Ting Wang ${ }^{1 \dagger}$, Junmei Zhang ${ }^{1}$, Xiang Zhou ${ }^{1}$, Yafan Niu ${ }^{1}$, Fu Liu ${ }^{1}$, \\ Yinan Zhong ${ }^{1 *}$, Dechun Huang ${ }^{1,2}$ and Wei Chen ${ }^{1,2 *}$ \\ ${ }^{1}$ Department of Pharmaceutical Engineering, School of Engineering, China Pharmaceutical University, Nanjing, China, \\ ${ }^{2}$ Engineering Research Center for Smart Pharmaceutical Manufacturing Technologies, Ministry of Education, School of \\ Engineering, China Pharmaceutical University, Nanjing, China
}

OPEN ACCESS

Edited by: Bin He,

Sichuan University, China

Reviewed by:

Yiran Zheng,

Soochow University, China

Xiaohui Pu,

Henan University, China

*Correspondence:

Yinan Zhong

ynzhong@cpu.edu.cn

Wei Chen

w.chen@cpu.edu.cn

${ }^{+}$These authors have contributed equally to this work

Specialty section: This article was submitted to Biomaterials,

a section of the journal

Frontiers in Bioengineering and

Biotechnology

Received: 30 September 2021

Accepted: 29 October 2021

Published: 29 November 2021

Citation:

Chen E, Wang T, Zhang J, Zhou X, Niu Y, Liu F, Zhong Y, Huang $D$ and Chen W (2021) Mitochondrial

Targeting and $\mathrm{pH}$-Responsive

Nanogels for Co-Delivery of Lonidamine and Paclitaxel to Conquer

Drug Resistance.

Front. Bioeng. Biotechnol. 9:787320.

doi: 10.3389/fbioe.2021.787320
Multidrug resistance (MDR) is one of the leading causes of the failure of cancer chemotherapy and mainly attributed to the overexpression of drug efflux transporters in cancer cells, which is dependent on adenosine triphosphate (ATP). To overcome this phenomenon, herein, a mitochondrial-directed $\mathrm{pH}$-sensitive polyvinyl alcohol (PVA) nanogel incorporating the hexokinase inhibitor lonidamine (LND) and the chemotherapeutic drug paclitaxel (PTX) was developed to restore the activity of PTX and synergistically treat drugresistant tumors. The introduction of 2-dimethylaminoethanethiol (DMA) moiety into the nanogels not only promoted the drug loading capacity but also enabled the lysosomal escape of the nanogels. The subsequent mitochondrial targeting facilitated the accumulation and acid-triggered payload release in the mitochondria. The released LND can destroy the mitochondria by exhausting the mitochondrial membrane potential (MMP), generating reactive oxygen species (ROS) and restraining the energy supply, resulting in apoptosis and susceptibility of the MCF-7/MDR cells to PTX. Hence, the nanogel-enabled combination regimen of $L N D$ and PTX showed a boosted anti-tumor efficacy in MCF-7/MDR cells. These mitochondrial-directed pH-sensitive PVA nanogels incorporating both PTX and LND represent a new nanoplatform for MDR reversal and enhanced therapeutic efficacy.

Keywords: multidrug resistance reversal, mitochondrial targeting, $\mathrm{pH}$ responsive, nanogel, combination therapy

\section{INTRODUCTION}

Chemotherapy remains one of the major means for treating tumors; however, the therapeutic efficacy is perplexed by many reasons including the multidrug resistance (MDR) (Lage 2008; Holohan et al., 2013; Rebucci and Michiels, 2013). The underlying mechanisms are complex and can be mainly summed up in the increased drug efflux from the cytoplasm to the extracellular compartment via the adenosine triphosphate (ATP)-binding cassette (ABC) transporters in the cells such as $\mathrm{P}$-glycoprotein (P-gP) and multidrug resistance-associated protein 1 (MRP1) driven by the energy supply of ATP (Cole 2014; Kartal-Yandim et al., 2016; Assaraf et al., 2019). In order to reverse MDR, many strategies have been dedicated to the MDR inhibition by interfering the energy supply, re-sensitizing the tumor cells to the chemotherapeutic drugs (Abdallah et al., 2015; Assanhou et al., 2015; Li et al., 2016; Tu et al., 2018; Gao et al., 2019; Cheng et al., 2021; Liu et al., 2021). 
Lonidamine (LND), which is a hexokinase inhibitor, blocks the energy supply and mitochondrial respiration, leading to mitochondrial dysfunction (Floridi et al., 1981; Price et al., 1996; Nath et al., 2016). In addition, LND triggers cell apoptosis by dissipating mitochondrial membrane potential (MMP) and generating reactive oxygen species (ROS). The combination of LND as a chemosensitizer with chemotherapeutic drugs provides an effective strategy for treating drug-resistant tumors (Li et al., 2002; Nath et al., 2013; Nath et al., 2015; Huang et al., 2020). Nevertheless, the poor water solubility and the lack of targetability of the drugs compromised their efficacy and limited their applications. Nanomedicine by virtue of its unique properties, such as the solubilization of the hydrophobic drugs and the delivery of the payload to the tumor sites via the enhanced permeability and retention (EPR) effect, has received widespread attention (Golombek et al., 2018; Majumder et al., 2019; Cao et al., 2020; Qiao et al., 2021). Nowadays, multifunctional nanomedicine integrating various modules of targeting (Qiao et al., 2019), lysosomal escaping, stimuli sensitivity, etc. (Pu et al., 2019; Zong et al., 2020; Zhong et al., 2021; Zong et al., 2021) could further improve the drug delivery efficacy, maximizing the therapeutic outcomes (Chen et al., 2017; Shahriari et al., 2019; Yang et al., 2019; He et al., 2021; Peng et al., 2021). It should be noted that the mitochondrial-targeting strategy plays an essential role in fully taking advantage of LND (Li et al., 2013; Zhang et al., 2015; Liu et al., 2017; Guo et al., 2021). Additionally, the biosafety of nanomedicine is a big concern.

In this study, we have constructed a triphenylphosphine (TPP)installed $\mathrm{pH}$-sensitive biocompatible polyvinyl alcohol (PVA) nanogel containing 2-dimethylaminoethanethiol (DMA) moiety for mitochondrial-directed co-delivery of LND and the chemotherapeutic drug paclitaxel (PTX) in drug-resistant tumors. The nanogels (T-D-NGs@LND and PTX) were fabricated by in situ crosslinking of the acrylate in PVA derivative under UV light and the concurrent co-loading of LND and PTX via hydrophobic and/or electrostatic interactions. After the internalization in the drug-resistant tumor cells, T-DNGs@LND and PTX escaped from the lysosome due to the proton sponge effect of DMA moiety, effectively accumulated in the mitochondria with the orientation of TPP module, and rapidly released the payload resulting from the acid-triggered cleavage of the acetal linker within the network of nanogels (Scheme 1). The unleashed LND acted on the mitochondria and de-energized the cancer cells, resulting in cell apoptosis and making the cells more susceptible to the released PTX. The nanogel-mediated drug resistance alleviation and synergistic effect significantly boosted the anti-tumor activity in MCF-7/MDR cells, holding a great potential for conquering MDR.

\section{EXPERIMENTAL SECTION}

\section{Preparation of DMA-Modified Vinyl Ether Acrylate-Functionalized PVA}

Vinyl ether acrylate (VEA) and VEA-functionalized PVA (PVA$g$-VEA) were prepared based on the previous reports (Chen et al., 2017). For the synthesis of DMA-functionalized PVA- $g$-VEA
(PVA- $g$-VEA-DMA), DMA and $\mathrm{Et}_{3} \mathrm{~N}(20 \mu \mathrm{l})$ were added to a 10 - $\mathrm{ml}$ solution of PVA- $g$-VEA $(0.413 \mathrm{~g}, 0.024 \mathrm{mmol})$ in methanol under stirring, and the reaction was continued for $12 \mathrm{~h}$. Afterwards, the above mixture solution was condensed by rotary evaporation. Finally, PVA-g-VEA-DMA was purified using the precipitant of ice-cold diethyl ether and then dried using the vacuum drier.

\section{Preparation of TPP-Functionalized PVA-g-VEA-DMA}

To synthesize TPP-modified PVA- $g$-VEA-DMA, a mixture of TPP (70.5 mg, $0.16 \mathrm{mmol})$, DCC (49.1 mg, $0.24 \mathrm{mmol}$ ), and DMAP (20.3 mg, $0.17 \mathrm{mmol})$ was dissolved in dimethyl sulfoxide (DMSO) $(10 \mathrm{ml})$, and the obtained solution was vigorously stirred for $1 \mathrm{~h}$ for activation of the carboxyl group in TPP. Subsequently, a solution of PVA- $g$-VEA-DMA (100 mg) in DMSO $(5 \mathrm{ml})$ was slowly added for a 24 -h reaction under stirring. After that, PVA-g-VEA-DMA/TPP was purified via extensive dialysis [molecular weight cut off (MWCO): $3.5 \mathrm{kDa}$ ] against methanol for $24 \mathrm{~h}$, concentrated through rotary evaporation, precipitated in ice-cold diethyl ether, as well as vacuum dried.

\section{Preparation and Characterization of Nanogels}

Acid-responsive nanogels were prepared using the UVcrosslinking method. Briefly, the nanogels were obtained by dissolving the polymer of PVA-g-VEA-DMA/TPP in water $(1 \mathrm{mg} / \mathrm{ml})$ with the addition of a photoinitiator (I2959, $5 \mathrm{wt} \%$ of polymer), adjusting solution $\mathrm{pH}$ to 6.8 , and stirring under $\mathrm{UV}$ exposure for $10 \mathrm{~min}$. To prepare drug-loaded nanogels, a solution of PTX and LND in DMSO $(10 \mathrm{mg} / \mathrm{ml})$ was added in the aqueous polymer solution with a feeding ratio of $10 \%$. Through adjusting the solution $\mathrm{pH}$ to 6.8 and in situ crosslinking of acrylate in the polymers under UV light for 10 min, PTX and LND co-loaded nanogels were obtained. A Millipore ultrafiltration centrifugal tube with a MWCO of 10,000 was used to remove the free drugs.

\section{pH-Induced Degradation of Nanogels and In Vitro Release Profile of LND and PTX}

$\mathrm{pH}$-induced degradation of nanogels was studied by detecting the nanogel size change via dynamic light scattering (DLS) in different conditions ( $\mathrm{pH} 7.4$ and 5.0) for different periods (0, 6 , and $24 \mathrm{~h}$ ). The unleash behavior of LND and PTX-loaded nanogels was explored in different conditions ( $\mathrm{pH} 7.4$ and 5.0) through the dialysis procedure. In brief, a dialysis bag (MWCO: 3,500 ) containing $1 \mathrm{ml}$ of PTX and LND co-loaded nanogel suspension was placed into a large-volume tube with $20 \mathrm{ml}$ of the corresponding medium under a continuous shaking at $100 \mathrm{rpm}$ at $37^{\circ} \mathrm{C}$. At various time intervals $(0,2,4,6,8,10,24$, 52,56 , and $72 \mathrm{~h}$ ), $5 \mathrm{ml}$ of the medium in the tube of each group was extracted and supplemented by the newly prepared medium. The collected media of each group were concentrated and subjected to HPLC for quantification of the released PTX and LND. 


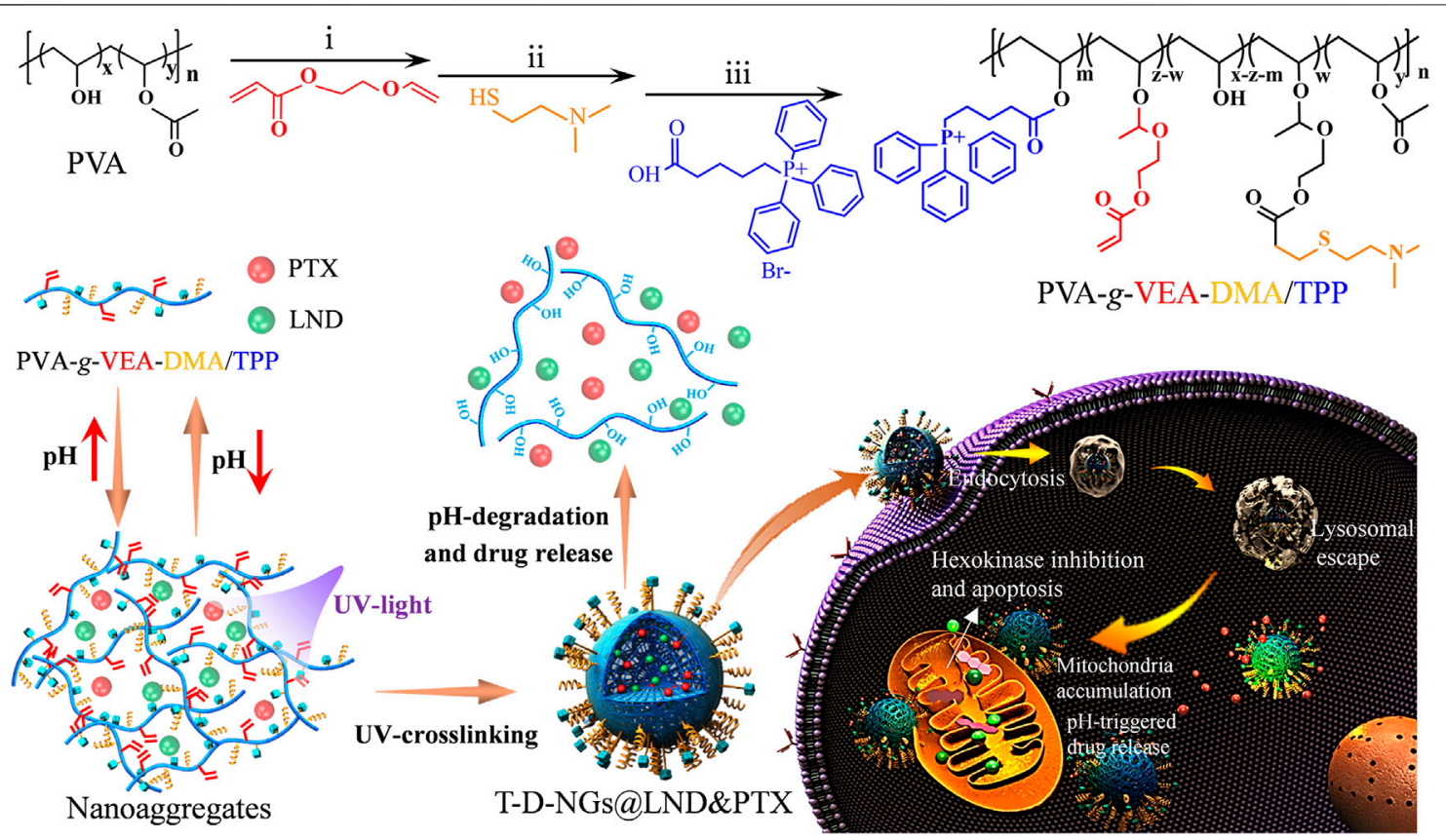

SCHEME 1 | Schematic illustrations for the constituent, fabrication, and destruction of T-D-NGs@LND and PTX and its endocytosis, lysosomal escape, mitochondrial orientation, and acid-triggered drug release within drug-resistant tumor cells for synergistic effect including LND-induced apoptosis, drug resistance alleviation, and restored chemotherapeutics of PTX.

\section{Lysosomal Escape and Co-Localization Into the Mitochondria}

MCF-7/MDR cells $\left(1 \times 10^{5}\right.$ cell/well $)$ were seeded into a 24 -well plate containing a rounded coverslip in each well. After $24 \mathrm{~h}$, fluorescein isothiocyanate (FITC)-labeled NGs, D-NGs, and T-D-NGs were added and incubated with the cells for $8 \mathrm{~h}$. Then, the medium was removed, and the cells were incubated with $500 \mu \mathrm{l}$ of serum-free media containing $200 \mathrm{nM}$ MitoTracker ${ }^{\circledR}$ Red CM$\mathrm{H} 2 \mathrm{XRos}$ probes at $37^{\circ} \mathrm{C}$ for $20 \mathrm{~min}$ or $500 \mu \mathrm{l}$ of serum-free media containing $50 \mathrm{nM}$ LysoTracker ${ }^{\circledR}$ Red DND-99 probes at $37^{\circ} \mathrm{C}$ for $30 \mathrm{~min}$. After washing thrice in phosphate-buffered saline (PBS) and fixation of the cells using 4\% paraformaldehyde for $20 \mathrm{~min}$, 4',6-diamidino-2-phenylindole (DAPI) was added for 10-min staining of the cell nucleus, and the excess DAPI was removed by washing thrice in PBS. Finally, the fluorescence images of each group were obtained by confocal laser microscope (CLSM, LSM700, Zeiss, Germany) and processed using the ZEN imaging software.

\section{Mitochondrial Membrane Potential $(\Delta \psi \mathrm{m})$ Depolarization}

To measure the mitochondrial depolarization, the cationic fluorochrome JC-1 was employed to detect mitochondrial membrane potential change. Briefly, MCF-7/MDR cells were seeded in a 12 -well plate $\left(1 \times 10^{5}\right.$ cells/well $)$ and incubated for 24 h. Then, free LND, NGs@LND,D-NGs@LND, and T-D-NGs@ LND at a LND dosage of $50 \mu \mathrm{g} / \mathrm{ml}$ were severally added and incubated with the cells for $12 \mathrm{~h}$. Thereafter, the cells were washed with PBS followed by incubation with $0.0125 \mathrm{M} \mathrm{JC}-1$ at $37^{\circ} \mathrm{C}$ for
20 min. After washing thrice in PBS, the cells were observed using a fluorescence microscope (IX73, Olympus, Japan).

\section{ROS Level Detection}

A ROS assay kit (Beyotime, China) was utilized for the detection of the intracellular ROS level. Briefly, MCF-7/MDR cells were cultured in a 12 -well plate $\left(1 \times 10^{5}\right.$ cells/well $)$ and incubated for $24 \mathrm{~h}$. Then, free LND, NGs@LND, D-NGs@LND, and T-D-NGs@LND at a LND dosage of $50 \mu \mathrm{g} / \mathrm{ml}$ were respectively added and incubated with the cells for $12 \mathrm{~h}$. Afterwards, the cells were washed with PBS for two times and stained with dichlorodihydro-fluorescein diacetate (DCFH-DA) for $20 \mathrm{~min}$. After washing thrice in PBS and fixation of the cells using 4\% paraformaldehyde for $20 \mathrm{~min}$, DAPI was added for a 10-min staining of the cell nucleus, and the excess DAPI was removed by washing thrice in PBS. Finally, the cells were observed using a fluorescence microscope (IX73, Olympus, Japan).

\section{ATP Content Detection}

An ATP assay kit (Beyotime, China) was utilized for the detection of the intracellular ATP levels. MCF-7/MDR cells were cultured in a six-well plate $\left(1 \times 10^{5}\right.$ cell/well $)$ for $24 \mathrm{~h}$. After that, free LND, NGs@LND, D-NGs@LND, and T-D-NGs@LND at a LND dosage of $50 \mu \mathrm{g} / \mathrm{ml}$ were respectively added and incubated with the cells for $12 \mathrm{~h}$. The intracellular ATP level was measured according to the manufacturer's protocol.

\section{In Vitro Anti-Tumor Activity}

To evaluate the apoptosis-inducing effect of different LND formulations, MCF-7/MDR cells were seeded into 12-well 


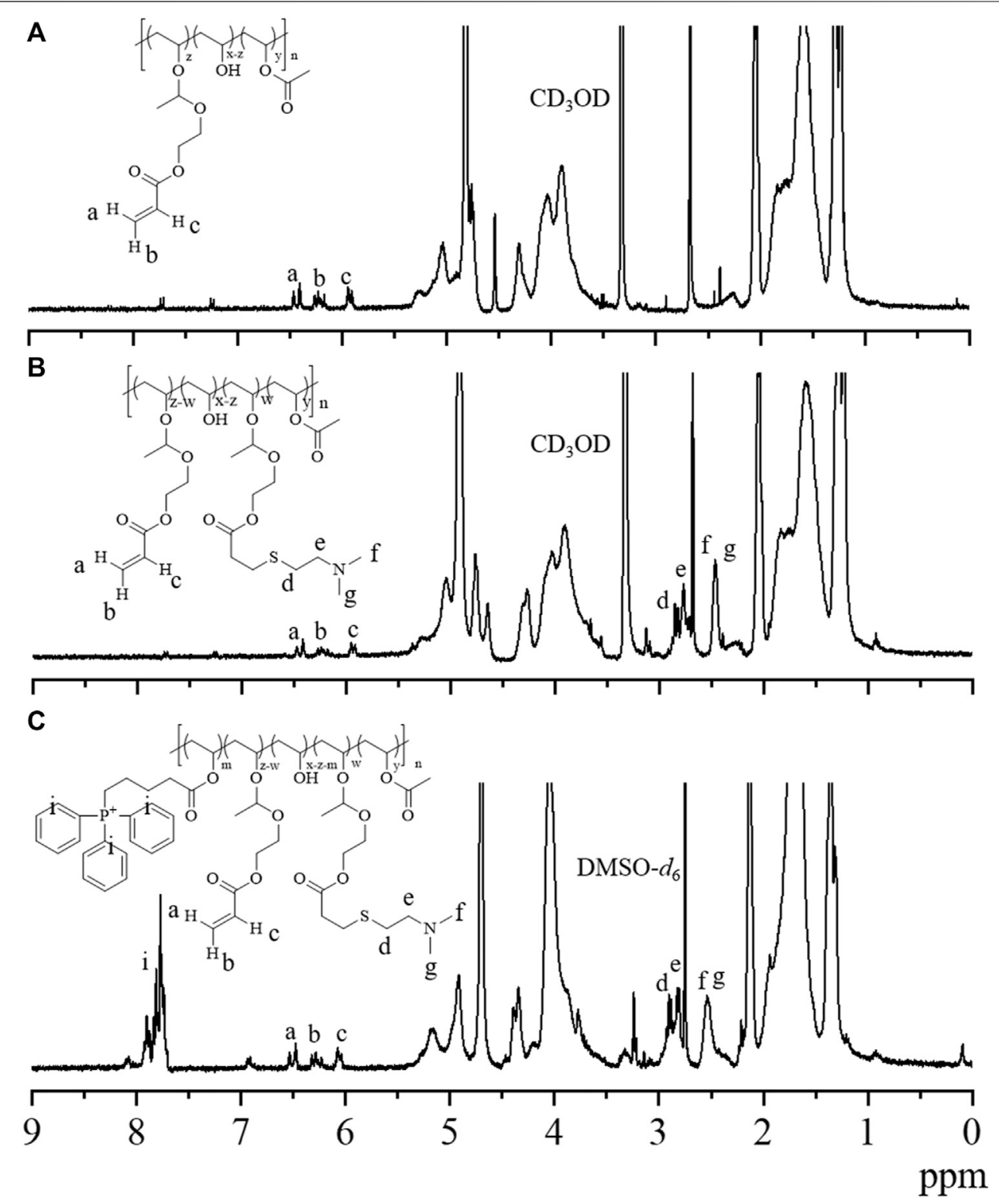

FIGURE 1| ${ }^{1} \mathrm{H}$ NMR spectra of (A) PVA-g-VEA (300 MHz, CD $\left.{ }_{3} \mathrm{OD}\right)$, (B) PVA-g-VEA-DMA (300 MHz, CD $\left.{ }_{3} \mathrm{OD}\right)$, and (C) PVA-g-VEA-DMATPP (300 MHz, DMSO$\left.d_{6}\right)$. VEA, vinyl ether acrylate; PVA-g-VEA, VEA-functionalized PVA; PVA-g-VEA-DMA, DMA-functionalized PVA-g-VEA; TPP, triphenylphosphine.

plates $\left(1 \times 10^{5}\right.$ cell/well $)$ and cultured for $24 \mathrm{~h}$ at $37^{\circ} \mathrm{C}$. Then, different LND formulations ( $50 \mu \mathrm{g} / \mathrm{ml} \mathrm{LND})$ were added and incubated with the cells for $12 \mathrm{~h}$. After that, the cells were digested and collected for Annexin V-FITC/propidium iodide (PI) staining based on the manufacturer's instructions and analyzed by flow cytometry.

MTT assay was utilized to study the cytotoxicity of MCF-7/ MDR cells receiving various formulations. The cells were cultured in 96-well plates for $24 \mathrm{~h}$ and then incubated with various formulations or free drug. After 48-h incubation, MTT solution was added for $4-\mathrm{h}$ incubation. Then, the culture medium was replaced by DMSO for detection under a microplate reader with the absorbance at $490 \mathrm{~nm}$.

\section{Statistical Analysis}

Data were expressed as mean \pm standard deviation. Differences between groups were assessed by a two-tailed unpaired Student's $t$-test or oneway ANOVA with Tukey's post hoc test. The level of significance was set at probabilities of ${ }^{*} p<0.05,{ }^{* *} p<0.01$, and ${ }^{* * *} p<0.001$.

\section{RESULTS AND DISCUSSION \\ Synthesis and Characterization of TPP-Modified PVA Nanogels}

We started with the synthesis of PVA- $g$-VEA/VEA-DMA/TPP polymer, in which the pendent VEA, positively charged DMA, 
and mitochondria-recognizable TPP were successively conjugated onto the water-soluble PVA backbone (Scheme 1). The VEA was firstly grafted onto PVA polymers via an acetalization reaction between vinyl ether and hydroxyl group in the presence of PTSA. The signals at $\delta 6.6$ and $\delta 5.85-6.45$ attributable to the vinyl protons of VEA and newly formed acetal methine proton, respectively, revealed the successful conjugation of VEA to the polymers, and the VEA grafting ratio was determined to be about $6.1 \%$ by comparing the integral of the peaks at $\delta 1.3$ and at $\delta 6.6$ (Figure 1A). DMA was further conjugated with acrylate in PVA-VEA, yielding a DMA functionality of about $2.66 \%$ with $3.44 \%$ acrylate left for UVmediated crosslinking according to the ${ }^{1} \mathrm{H}$ NMR spectra (Figure 1B). Lastly, the obtained polymeric conjugates were coupled with TPP on the backbone using DCC and DMAP via esterification, and the content of the TPP moiety on PVAVEA-DMA-TPP was about $1.3 \mathrm{wt} \%$ as revealed from the integrals of the peaks at $\delta 7.68$ of benzene protons and at $\delta 1.3$ (Figure 1C).

T-D-NGs were then obtained by dissolving the polymer of PVA- $g$-VEA/VEA-DMA/TPP in water, adjusting $\mathrm{pH}$ to 7.8, crosslinking acrylate in the presence of I2959 under UV light. D-NGs and NGs were prepared in a similar way. As displayed in Figure 2A-C, T-D-NGs, D-NGs, and NGs presented a typical spherical shape with uniform sizes of about $170 \mathrm{~nm} . \mathrm{pH}-$ responsive T-D-NGs were explored by detecting the size change of T-D-NGs in acetate buffer ( $\mathrm{pH}$ 5.0) for different times via DLS measurement. T-D-NGs displayed a swelled structure with a size of about several hundred nanometers after $6 \mathrm{~h}$ and later a small-sized particle with a size of about $17 \mathrm{~nm}$ after $24 \mathrm{~h}$, whereas the size of T-D-NGs maintained almost the same at $\mathrm{pH} 7.4$ after 24-h incubation (Figure 2D). These results revealed that $\mathrm{T}$-D-NGs possessed a good colloidal stability in the physiological condition while suffered from the destruction at $\mathrm{pH} 5.0$ that mimicked intracellular acidic condition. It should be noted that the swelled structure of T-D-NGs in response to acidic condition in their initial stage was ascribed to their partial cleavage of the acetal linkers in the network of T-D-NGs, which ultimately led to complete disassociation and small-sized conjugates in the acidic condition.

\section{Preparation and Characterization of Drug-Loaded Nanogels}

LND, which is a small-molecule hexokinase inhibitor, and chemotherapeutic PTX were co-loaded into the T-D-NGs via hydrophobic and/or electrostatic interactions. LND and PTXloaded nanogels showed increased sizes in comparison to the blank nanogels as determined by DLS (Table 1). The introduction of DMA and TPP moieties in the nanogels dramatically improved drug loading content (DLC) from $2 \mathrm{wt} \%$ to about $5 \mathrm{wt} \%$ at a theoretical DLC of $10 \mathrm{wt} \%$, which was probably due to their increased hydrophobicity and electropositivity.

The in vitro LND and PTX release profiles from T-D-NGs were studied at various $\mathrm{pH}$, i.e., $\mathrm{pH} 5.0$ and $\mathrm{pH}$ 7.4. As displayed in Figure 2E, F, a small amount of LND (31.7\%) and PTX (34.5\%) was leaked from T-D-NGs at $\mathrm{pH} 7.4$ after $72 \mathrm{~h}$, respectively, whereas more than $70 \%$ of LND and PTX were released at pH 5.0 after $72 \mathrm{~h}$, further confirming $\mathrm{pH}$-triggered disassociation of the nanogels and the subsequent payload release.

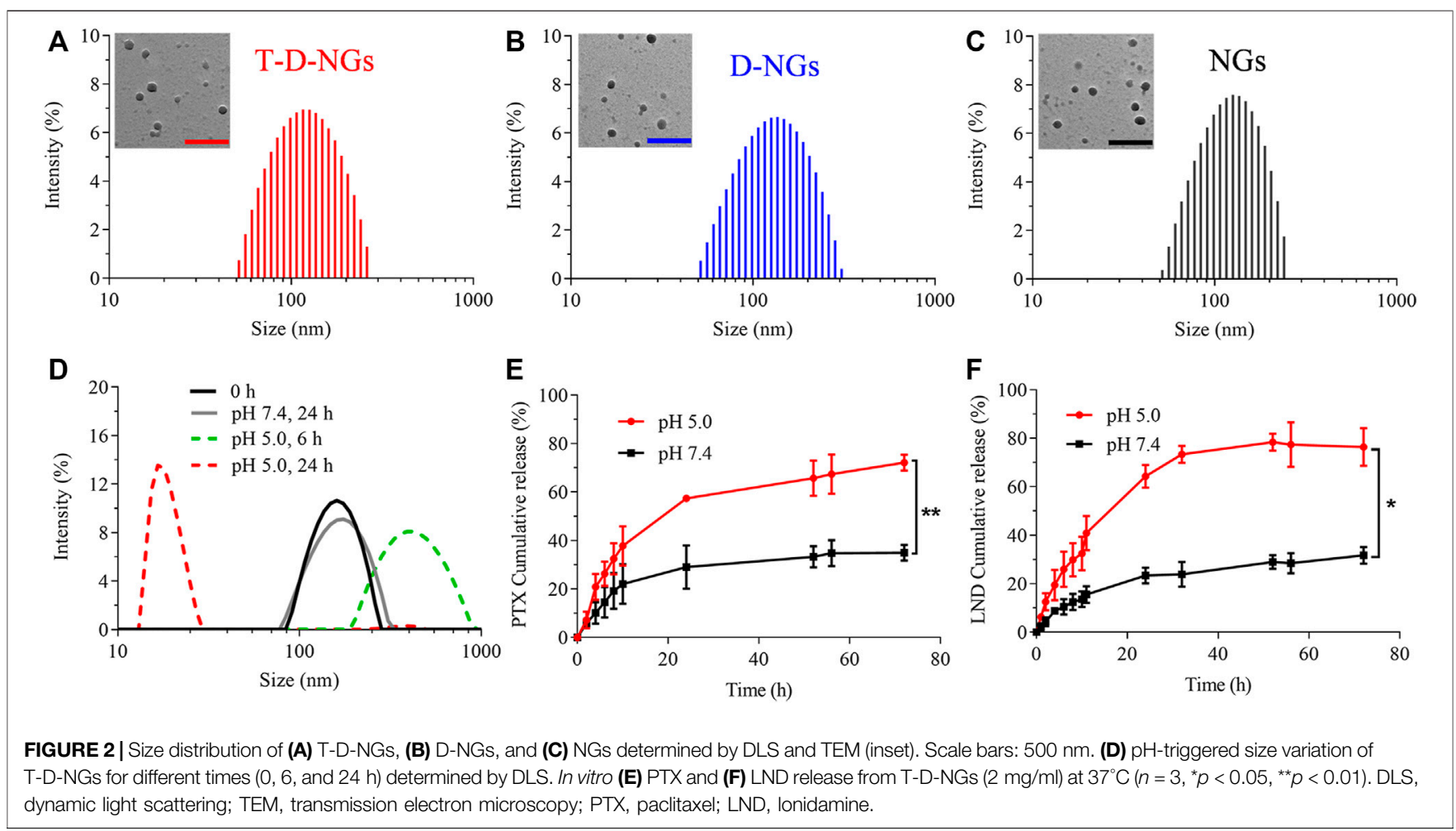


TABLE 1 | Characteristics of different drug-loaded nanogels ${ }^{a}$

\begin{tabular}{|c|c|c|c|c|c|c|}
\hline \multirow[t]{2}{*}{ Nanogels } & \multicolumn{2}{|c|}{ DLE $(\%)^{\mathrm{b}}$} & \multicolumn{2}{|c|}{ DLC $(\%)^{b}$} & \multirow[t]{2}{*}{ Size $^{c}$} & \multirow[t]{2}{*}{ PDI $^{\circ}$} \\
\hline & PTX & LND & PTX & LND & & \\
\hline NGs & $23.01 \pm 1.0$ & $20.74 \pm 0.2$ & $2.25 \pm 0.1$ & $2.03 \pm 0.1$ & $165.6 \pm 0.6$ & 0.22 \\
\hline D-NGs & $46.70 \pm 5.5$ & $57.69 \pm 6.7$ & $4.46 \pm 0.5$ & $5.45 \pm 0.6$ & $211.7 \pm 3.2$ & 0.15 \\
\hline T-D-NG & $53.21 \pm 0.9$ & $74.92 \pm 0.4$ & $5.05 \pm 0.1$ & $5.05 \pm 0.1$ & $182.3 \pm 6.1$ & 0.21 \\
\hline
\end{tabular}

${ }^{a}$ The concentration of nanogels was set at $1.0 \mathrm{mg} / \mathrm{ml}$.

${ }^{b}$ Determined by HPLC.

${ }^{c}$ Determined by DLS.

\section{Escape From Lysosomes and Mitochondrial Targeting}

The cellular behaviors of T-D-NGs were investigated by using CLSM in MCF-7/MDR cells. T-D-NGs, D-NGs, and NGs were labeled with FITC for CLSM imaging, and the results showed that FITC-labeled NGs (FITC-NGs, green signal) were captured by lysosomes (red signal) with colocalized orange signal within cells for 8-h incubation, while most of the green signal of FITClabeled T-D-NGs (FITC-T-D-NGs) and FITC-labeled D-NGs (FITC-D-NGs) were separated from the red signal of lysosomes after $8 \mathrm{~h}$, indicating the successful escape of FITC-T-D-NGs and FITC-D-NGs from lysosomes with the aid of DMA module in the nanogels (Figure 3). In the following, we evaluated the mitochondrial-targeting ability of FITC-T-D-NGs, FITC-DNGs, and FITC-NGs by staining the mitochondria with MitoTracker (red). After $12 \mathrm{~h}$, a large area of FITC-T-D-NGs were overlapped with the mitochondria, resulting in intense orange fluorescence in the merged image (Figure 4). In contrast, FITC-D-NGs presented separated green and red fluorescence, and hardly any green signal was observed in the FITC-NG group with only red fluorescence in the merged image. All the above results demonstrated that FITC-T-D-NGs could efficiently escape from the lysosomes and target the mitochondria for facilitating LND delivery, while FITC-NGs and FITC-D-NGs either got trapped in the lysosomes or lacked mitochondrial orientation.

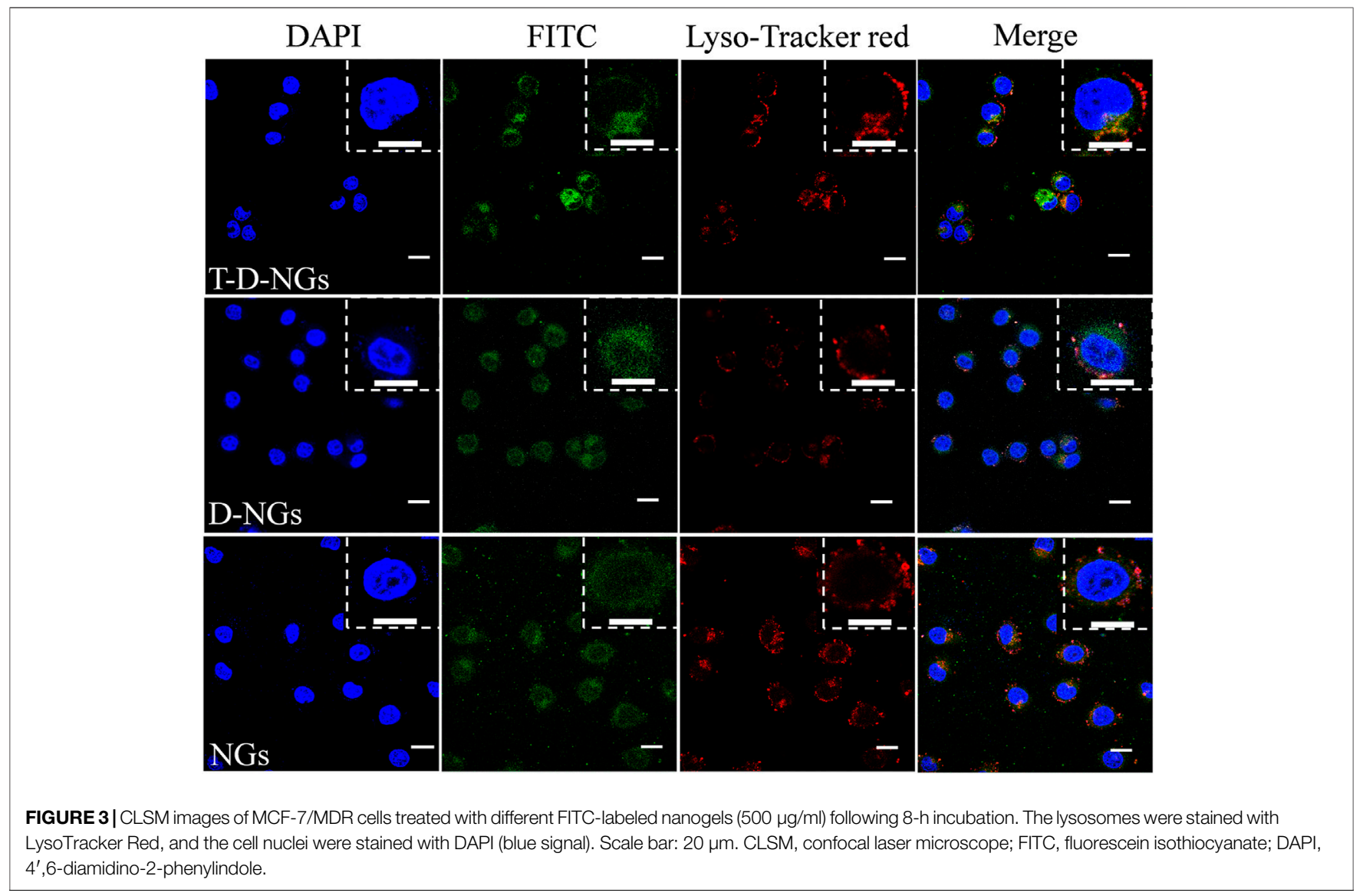




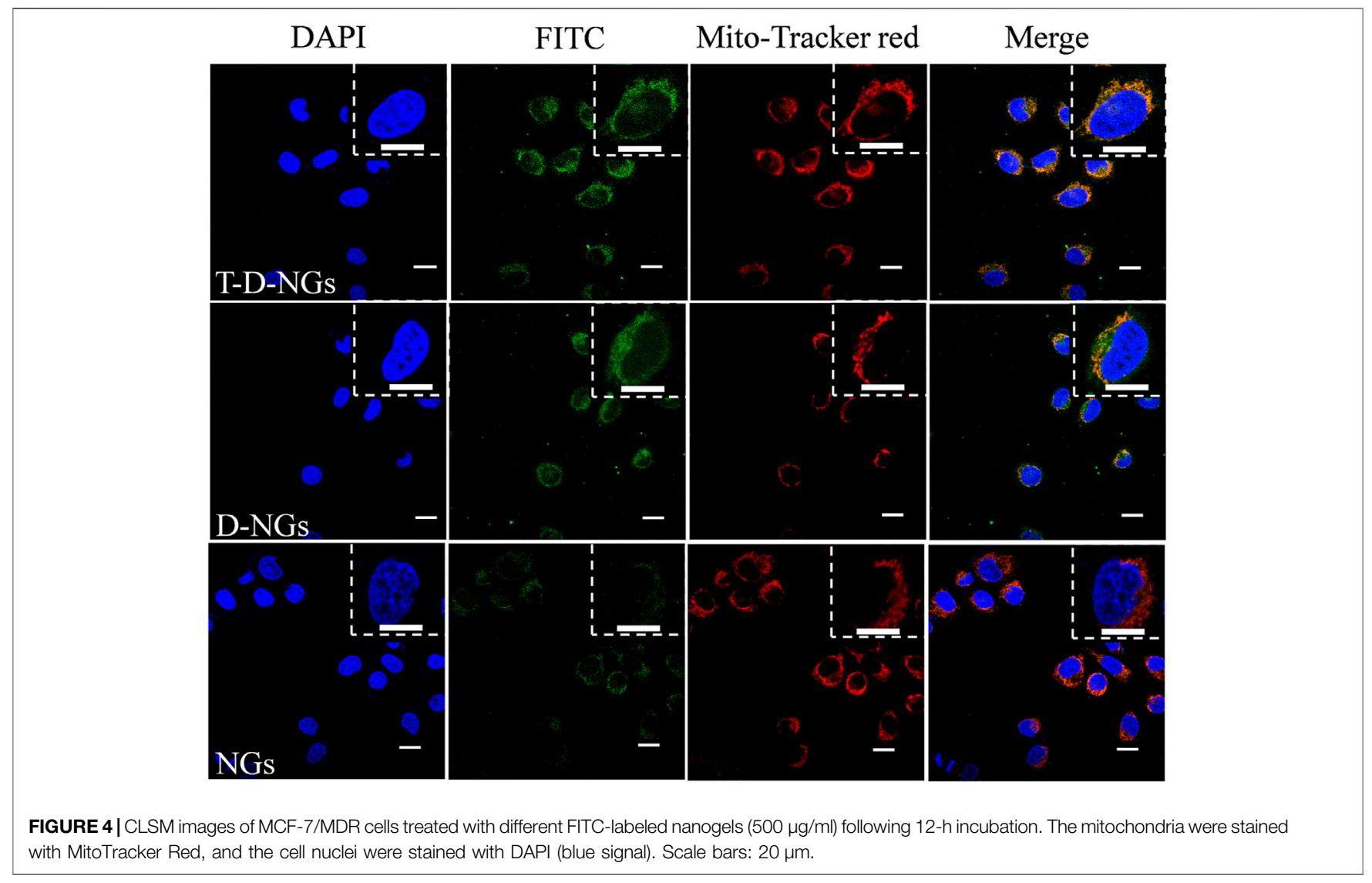

\section{Dysfunction of the Mitochondria}

To explore the underlying mechanism of LND-loaded T-D-NGs (T-D-NGs@LND) in interfering mitochondrial functions, the measurements of MMP, ROS production, and ATP secretion in MCF-7/MDR cells were performed. JC-1 staining was firstly employed, which served as a fluorescence probe to detect MMP. JC-1 forms aggregates with red fluorescence at a high MMP, while remains monomers with green fluorescence at a low potential level, which is indicative of early apoptosis. After 12-h incubation, free LND displayed minimal green signal and intense red signal level comparable to the PBS group in MCF-7/MDR cells due to its slow diffusion and poor mitochondrial targeting (Figure 5A; Supplementary Figure S1). NGs@LND slightly increased the green fluorescence to some extent and maintained the strong red fluorescence, revealing an insufficient dissipation of MMP because of being trapped in lysosomes and limited accumulation in the mitochondria as demonstrated above. D-NGs@LND ameliorated this phenomena because of its lysosomal escape aided by DMA moieties in the nanogels. T-D-NGs@LND was the most effective in dissipating MMP with the strongest green fluorescence concomitant with the weakest red fluorescence, which was ascribed to its lysosomal escape, mitochondrial accumulation, and effective LND release triggered by acid within cells.

ROS production causes oxidative cell damage at a high level, which was another mechanism involved in cancer cell apoptosis via the mitochondrial pathway. ROS production level was detected by a fluorescence probe of DCFH-DA via CLSM imaging. The trend of the intracellular ROS production level was similar with the results of the dissipation of MMP (Figure 6; Supplementary Figure S2), verifying that T-D-NGs@LND induced a large amount of intracellular ROS production in the mitochondria, potentially for anti-tumor applications.

Cumulative evidences demonstrated that LND had the potential to inhibit hexokinase enzyme that was implicated in ATP generation and mitochondrial respiration. ATP secretion from the MCF-7/MDR cells receiving various LND formulations was estimated through the ATP assay kit. As expected, T-DNGs@LND significantly decreased ATP secretion from the cells with a decline of about $60.9 \%$ versus $43.48 \%, 30.44 \%$, and $15.95 \%$ for D-NGs@LND, NGs@LND, and free LND severally after 12-h incubation (Supplementary Figure S3). Such a pronounced inhibition of ATP secretion in MCF-7/MDR cell induced by T-D-NGs@LND further confirmed the precise and efficient delivery of LND into the mitochondria and also laid a solid foundation for MDR reversal by suppressing P-gp that was dependent on ATP.

\section{Anti-Tumor Activity}

The cell apoptosis with the treatments of various LND formulations via the mitochondrial pathway was explored via Annexin V-FITC/PI staining through flow cytometry. LND 
A Monomer
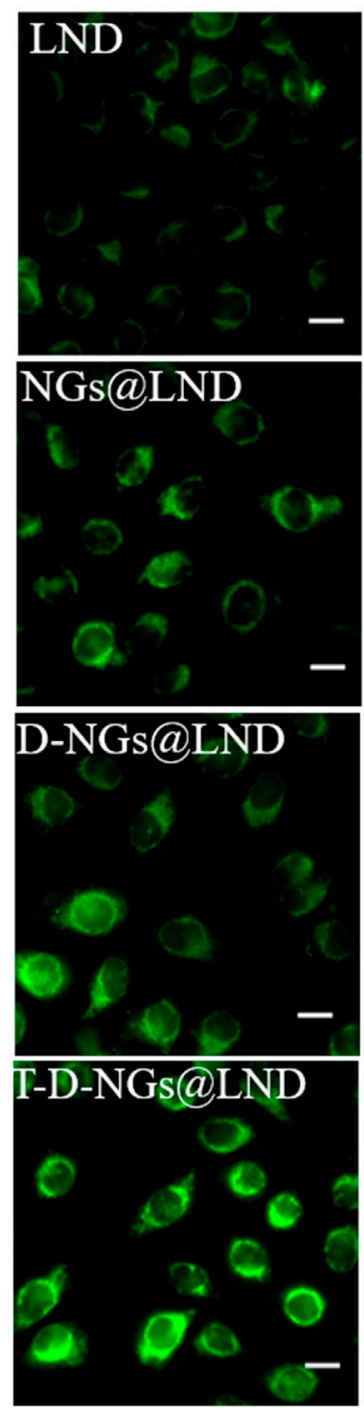

Aggregate
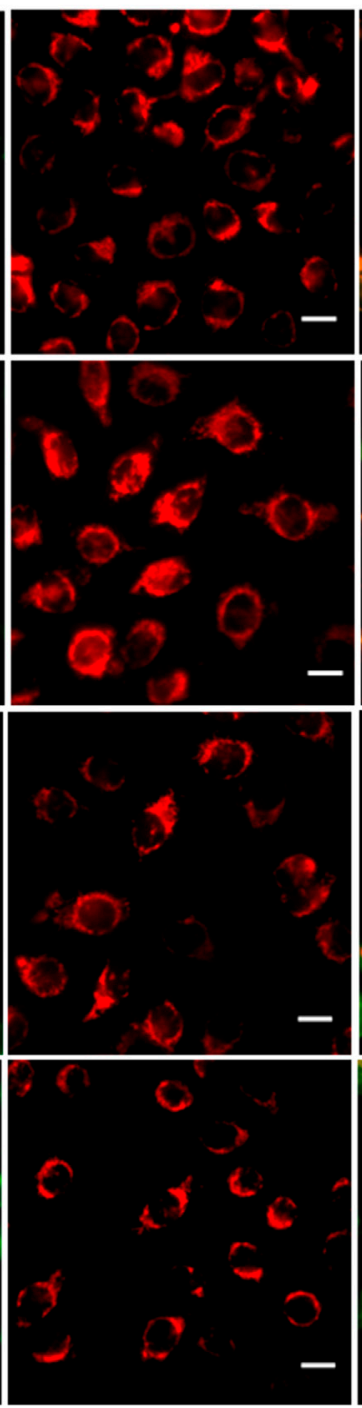

Merge
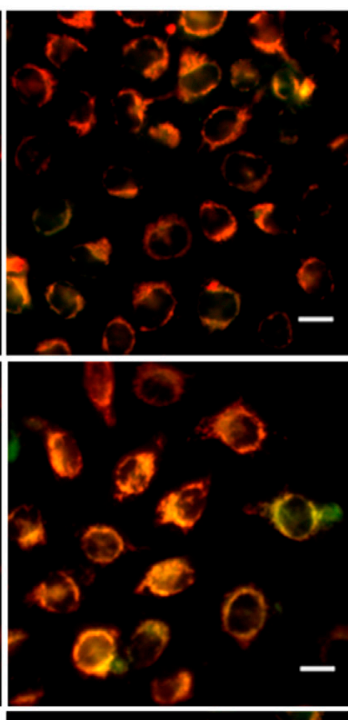

B Apoptosis
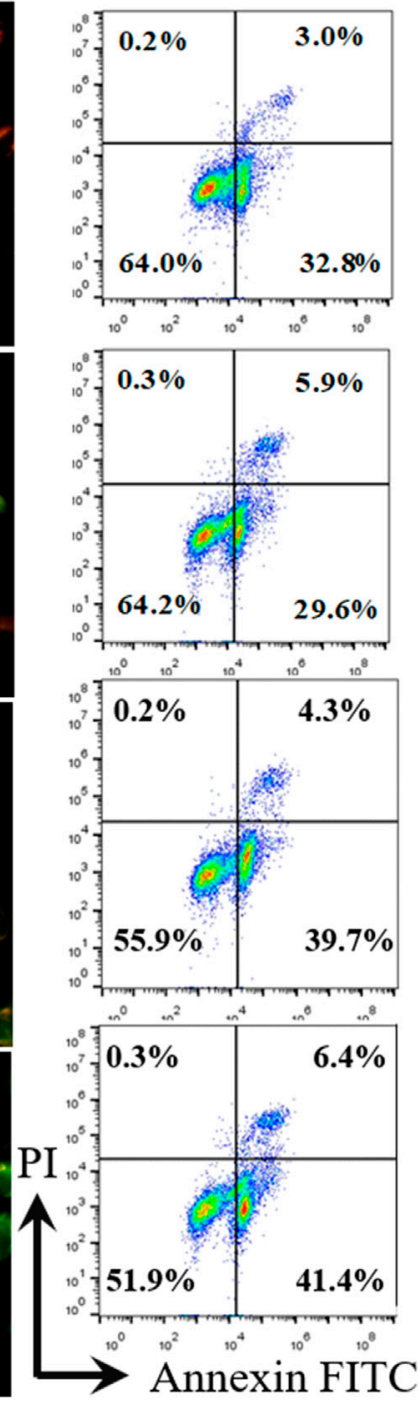

FIGURE 5 | (A) The MMP depolarization level of MCF-7/MDR cells incubated with LND, NGs@LND, D-NGs@LND, and T-D-NGs@LND for 12 h. The mitochondrial channel was stained with JC-1. Scale bars: $20 \mu \mathrm{m}$. (B) Cell apoptosis studies with treatments of various LND-loaded nanogels for 12 h. MMP, mitochondrial membrane potential.

could induce cell apoptosis via several mechanisms like destruction of MMP and oxidative stress of overproduced ROS in the mitochondria as noted above. Total apoptotic rates of about 47.8\% was found in T-D-NGs@LND-treated cells, which was higher than the D-NGs@LND (44\%), NGs@LND (35.5\%), and free LND (35.8\%) groups (Figure 5B). Moreover, T-D-NGs@LND caused both higher early (41.4\%) and late (6.4\%) apoptosis than other treatments. MTT assay was also carried out and showed that all of the LND formulations led to a dose-dependent cytotoxicity toward MCF-7/MDR cells, among which T-D-NGs@LND displayed the most potent anti-tumor activity with a cell viability of about $33.47 \%$ at a LND concentration of $100 \mu \mathrm{g} / \mathrm{ml}$ for $48 \mathrm{~h}$ (Figure 7A). Taken together, T-D-NGs@LND was demonstrated to exert direct tumor cell-killing ability of LND by overcoming lysosomal trap, navigating into the mitochondria and efficiently releasing LND.

Furthermore, the potential role of LND for drug resistance reversal and chemo-sensitization was utilized to reactivate the treatment ability of PTX in MCF-7/MDR cells. Thus, the combination of LND and PTX in T-D-NGs could bring about a synergistic anti-tumor activity by fully exerting tumor cell killing of both LND and PTX. To verify the above assumption, MTT assay was performed in MCF-7/MDR cells receiving PTX- and/or LNDloaded nanogels for $48 \mathrm{~h}$. As displayed in Figure 7B, all the PTX formulations and free PTX revealed mild cytotoxicity toward the cells with cell viabilities more than $40 \%$ even at a high PTX 


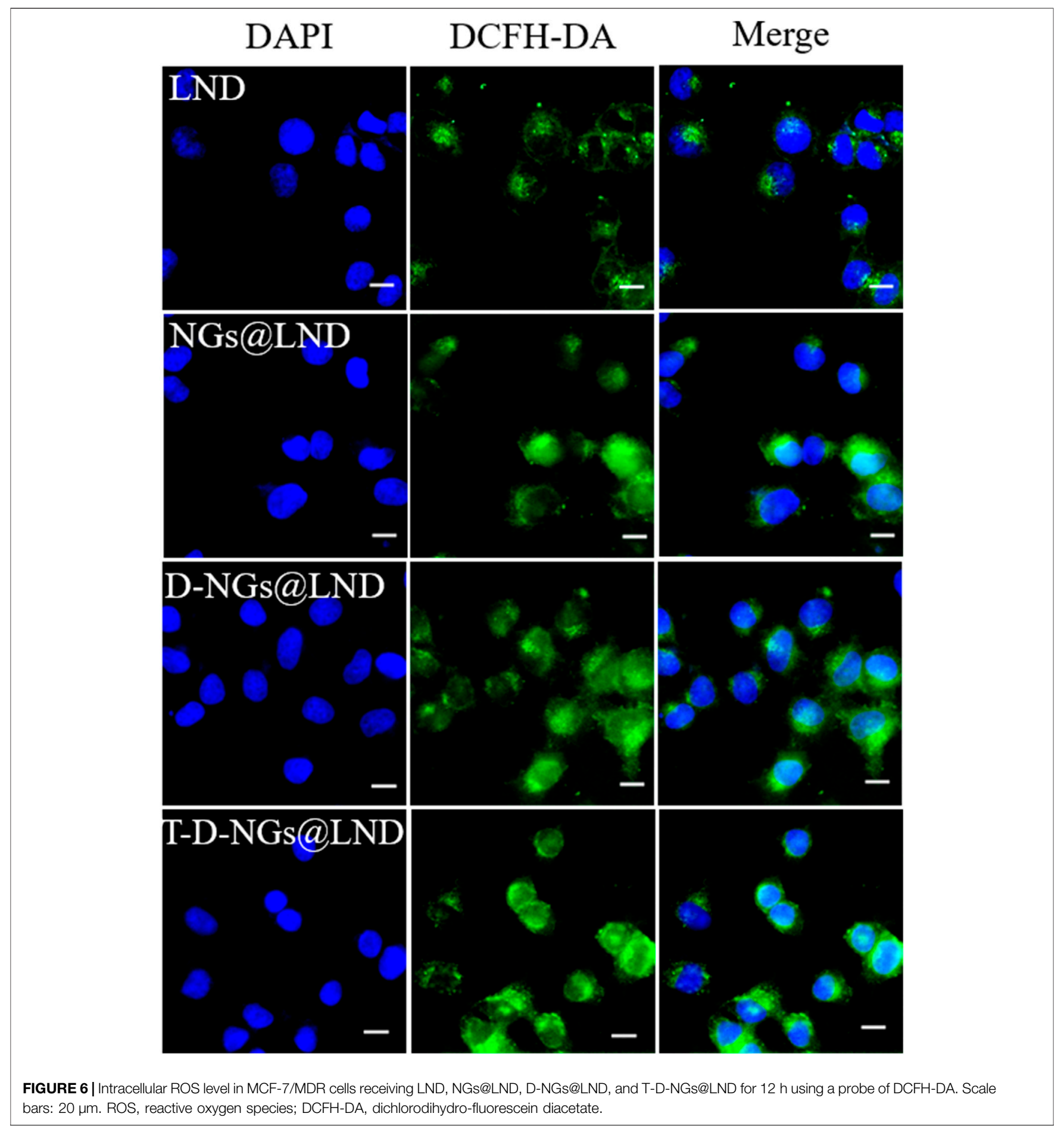

concentration of $20 \mu \mathrm{g} / \mathrm{ml}$, reflecting drug resistance of the cells and inactivation of PTX formulations. The combination of LND and PTX in various formulations showed cytotoxicity toward the cells in a PTX dose-dependent fashion at a fixed LND concentration of $50 \mu \mathrm{g} / \mathrm{ml}$ (Figure 7C). As predicted, T-DNGs@LND and PTX remarkably boosted the tumor cell-killing activity with a cell viability of about $18.59 \%$ at LND and PTX concentrations of 50 and $20 \mu \mathrm{g} / \mathrm{ml}$ for $48 \mathrm{~h}$, which was much lower than T-D-NG-enabled monotherapy (T-D-NGs@LND and T-DNGs@PTX), revealing the reversal of drug resistance and the synergistic therapeutic efficacy of unleashed LND and PTX. The half maximal inhibitory PTX concentration $\left(\mathrm{IC}_{50}\right)$ of T-D-NGs@ LND and PTX (LND: $50 \mu \mathrm{g} / \mathrm{ml}$ ) was also calculated, which was determined to be $7.44 \mu \mathrm{g} / \mathrm{ml}$. T-D-NGs@LND and PTX also displayed a much higher anti-tumor activity as compared with D-NGs@LND and PTX ( IC $\left._{50}: 10.70 \mu \mathrm{g} / \mathrm{ml}\right)$, NGs@LND and PTX 

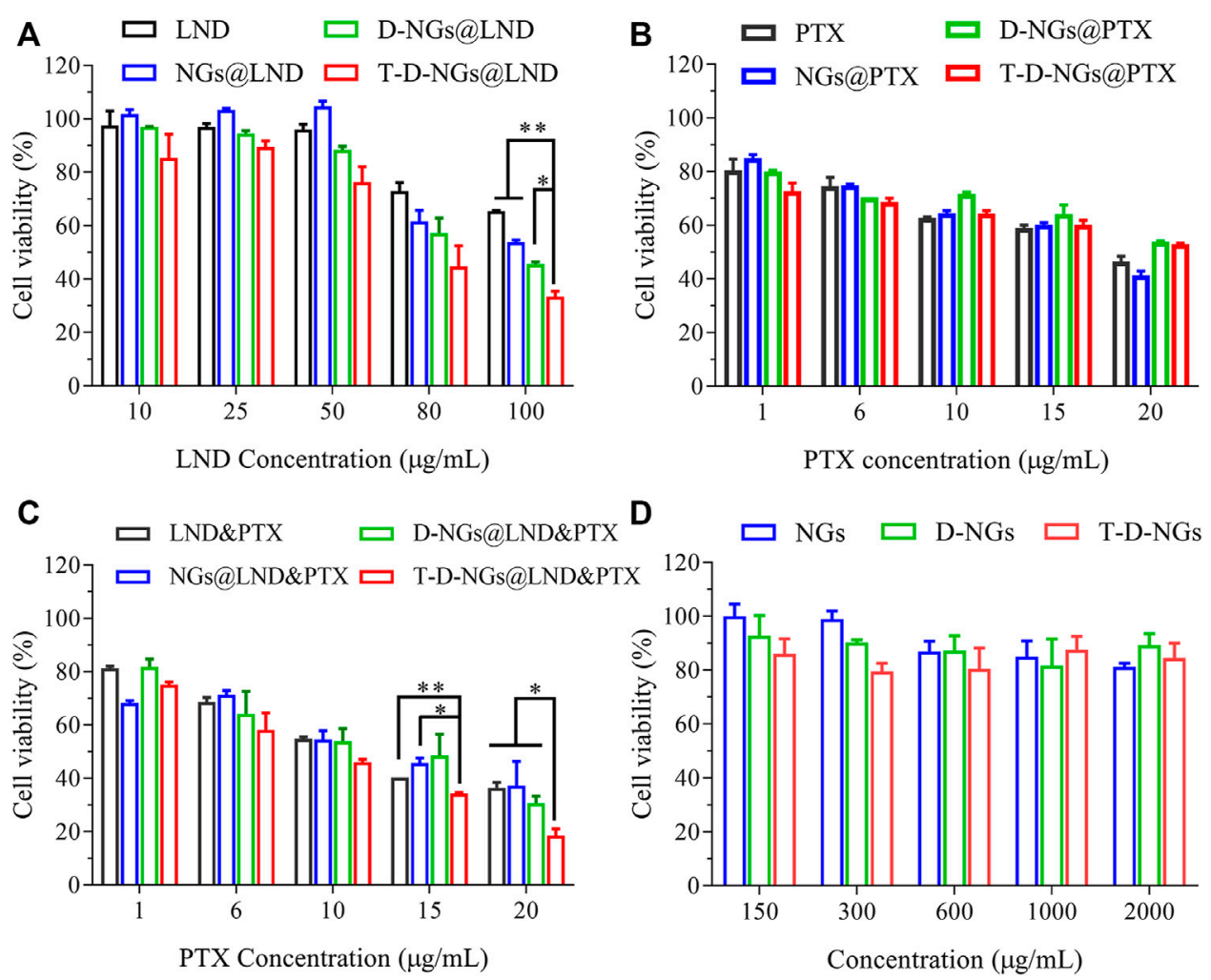

FIGURE 7 | Cytotoxicity of MCF-7/MDR cells after 48-h treatments of (A) LND-loaded nanogels, (B) PTX-loaded nanogels, (C) LND and PTX-loaded nanogels (LND: $50 \mu \mathrm{g} / \mathrm{ml})$, and (D) drug-free nanogels $\left(n=3,{ }^{*} p<0.05,{ }^{* *} p<0.01\right)$.

$\left(\mathrm{IC}_{50}: 11.96 \mu \mathrm{g} / \mathrm{ml}\right)$, and LND and PTX $\left(\mathrm{IC}_{50}: 11.49 \mu \mathrm{g} / \mathrm{ml}\right)$, further verifying T-D-NG-mediated lysosomal escape, mitochondrial accumulation, and acid-triggered payload release for fully exerting the efficacy of both drugs. In the meanwhile, the cytotoxicities of drug-free nanogels toward the cells were also assessed, and the results showed cell viabilities of $80 \%-100 \%$ at nanogel concentrations ranging from 0.15 to $2 \mathrm{mg} / \mathrm{ml}$ (Figure 7D), confirming the superior biocompatibility of the nanogels, which was mainly made up of FDA-approved biocompatible PVA backbone.

\section{CONCLUSION}

In conclusion, we have developed mitochondrial-directed $\mathrm{pH}$ sensitive nanogels incorporating LND and PTX (T-D-NGs@ LND and PTX) for drug resistance reversal and synergistic therapy against drug-resistant tumors. After internalization into MCF-7/MDR cells, T-D-NGs@LND and PTX efficiently escaped from the lysosomes with the aid of DMA moiety in the nanogels, accumulated into the mitochondria, and rapidly released the payload in the acidic conditions. The released LND, on one hand, directly induced cell apoptosis via dampening the mitochondrial membrane potential, promoting oxidative stress from the ROS production; on the other hand, it facilitated drug resistance reversal and sensitization of PTX activity by blocking the energy source and thus inhibiting drug resistance-related P-gp expression. As a result, T-D-NGs@ LND and PTX demonstrated a significantly potent anti-tumor activity against MCF-7/MDR cells with an extremely low $\mathrm{IC}_{50}$ of $7.44 \mu \mathrm{g} / \mathrm{ml}$ PTX (LND: $50 \mu \mathrm{g} / \mathrm{ml}$ ) after $48-\mathrm{h}$ treatment and a synergistic effect of LND and PTX. Our work provides a promising and synergistic strategy to conquer tumor MDR.

\section{DATA AVAILABILITY STATEMENT}

The original contributions presented in the study are included in the article/Supplementary Material; further inquiries can be directed to the corresponding authors.

\section{AUTHOR CONTRIBUTIONS}

EC conducted the investigation, formal analysis, and original draft preparation. TW conducted the data curation, methodology, and formal analysis and reviewed and edited the manuscript. JZ conducted the data curation and reviewed and edited the manuscript. XZ conducted the data curation, investigation, and software processing. YN and FL conducted the investigation. YZ supervised the study, acquired funding, served as project administrator, and reviewed and edited the manuscript. DH contributed resources and acquired funding. 
WC supervised and conceptualized the study, served as project administrator, acquired funding, and reviewed and edited the manuscript. All authors contributed to the article and approved the submitted version.

\section{FUNDING}

This work was funded by the National Natural Science Foundation of China (NSFC 51803238, 51973233, and

\section{REFERENCES}

Abdallah, H. M., Al-Abd, A. M., El-Dine, R. S., and El-Halawany, A. M. (2015). P-glycoprotein Inhibitors of Natural Origin as Potential Tumor ChemoSensitizers: A Review. J. Adv. Res. 6, 45-62. doi:10.1016/j.jare.2014.11.008

Assanhou, A. G., Li, W., Zhang, L., Xue, L., Kong, L., Sun, H., et al. (2015). Reversal of Multidrug Resistance by Co-delivery of Paclitaxel and Lonidamine Using a Tpgs and Hyaluronic Acid Dual-Functionalized Liposome for Cancer Treatment. Biomaterials 73, 284-295. doi:10.1016/j.biomaterials.2015.09.022

Assaraf, Y. G., Brozovic, A., Gonçalves, A. C., Jurkovicova, D., Linē, A., Machuqueiro, M., et al. (2019). The Multi-Factorial Nature of Clinical Multidrug Resistance in Cancer. Drug Resist. Updates 46, 100645. doi:10.1016/j.drup.2019.100645

Cao, J., Huang, D., and Peppas, N. A. (2020). Advanced Engineered Nanoparticulate Platforms to Address Key Biological Barriers for Delivering Chemotherapeutic Agents to Target Sites. Adv. Drug Deliv. Rev. 167, 170-188. doi:10.1016/j.addr.2020.06.030

Chen, W., Hou, Y., Tu, Z., Gao, L., and Haag, R. (2017). Ph-degradable Pva-Based Nanogels via Photo-Crosslinking of Thermo-Preinduced Nanoaggregates for Controlled Drug Delivery. J. Controlled Release 259, 160-167. doi:10.1016/ j.jconrel.2016.10.032

Cheng, F., Pan, Q., Gao, W., Pu, Y., Luo, K., and He, B. (2021). Reversing Chemotherapy Resistance by a Synergy between Lysosomal Ph-Activated Mitochondrial Drug Delivery and Erlotinib-Mediated Drug Efflux Inhibition. ACS Appl. Mater. Inter. 13, 29257-29268. doi:10.1021/acsami.1c03196

Cole, S. P. C. (2014). Multidrug Resistance Protein 1 (Mrp1, Abcc1), a "multitasking" Atp-Binding Cassette (Abc) Transporter. J. Biol. Chem. 289, 30880-30888. doi:10.1074/jbc.R114.609248

Floridi, A., Paggi, M. G., Marcante, M. L., Silvestrini, B., Caputo, A., and De Martino, C. (1981). Lonidamine, a Selective Inhibitor of Aerobic Glycolysis of Murine Tumor Cells. J. Natl. Cancer Inst. 66, 497-499. doi:10.1093/jnci/ 66.3.497

Gao, L., Dong, B., Zhang, J., Chen, Y., Qiao, H., Liu, Z., et al. (2019). Functional Biodegradable Nitric Oxide Donor-Containing Polycarbonate-Based Micelles for Reduction-Triggered Drug Release and Overcoming Multidrug Resistance. ACS Macro Lett. 8, 1552-1558. doi:10.1021/ acsmacrolett.9b00758

Golombek, S. K., May, J.-N., Theek, B., Appold, L., Drude, N., Kiessling, F., et al. (2018). Tumor Targeting via Epr: Strategies to Enhance Patient Responses. Adv. Drug Deliv. Rev. 130, 17-38. doi:10.1016/j.addr.2018.07.007

Guo, X., Yang, N., Ji, W., Zhang, H., Dong, X., Zhou, Z., et al. (2021). Mito-Bomb: Targeting Mitochondria for Cancer Therapy. Adv. Mater. 33, 2007778. doi:10.1002/adma.202007778

He, Y., Lei, L., Cao, J., Yang, X., Cai, S., Tong, F., et al. (2021). A Combinational Chemo-Immune Therapy Using an Enzyme-Sensitive Nanoplatform for DualDrug Delivery to Specific Sites by cascade Targeting. Sci. Adv. 7, 1. doi:10.1126/ sciadv.aba0776

Holohan, C., Van Schaeybroeck, S., Longley, D. B., and Johnston, P. G. (2013). Cancer Drug Resistance: An Evolving Paradigm. Nat. Rev. Cancer 13, 714-726. doi:10.1038/nrc3599

Huang, Y., Sun, G., Sun, X., Li, F., Zhao, L., Zhong, R., et al. (2020). The Potential of Lonidamine in Combination with Chemotherapy and Physical Therapy in Cancer Treatment. Cancers 12, 3332. doi:10.3390/cancers 12113332
21878337), the Jiangsu Specially-Appointed Professor Program, and the Nanjing Scientific and Technological Innovation Program for Overseas Talents to WC.

\section{SUPPLEMENTARY MATERIAL}

The Supplementary Material for this article can be found online at: https://www.frontiersin.org/articles/10.3389/fbioe.2021.787320/ full\#supplementary-material.

Kartal-Yandim, M., Adan-Gokbulut, A., and Baran, Y. (2016). Molecular Mechanisms of Drug Resistance and its Reversal in Cancer. Crit. Rev. Biotechnol. 36, 716-726. doi:10.3109/07388551.2015.1015957

Lage, H. (2008). An Overview of Cancer Multidrug Resistance: A Still Unsolved Problem. Cell. Mol. Life Sci. 65, 3145-3167. doi:10.1007/s00018-008-8111-5

Li, N., Zhang, C.-X., Wang, X.-X., Zhang, L., Ma, X., Zhou, J., et al. (2013). Development of Targeting Lonidamine Liposomes that Circumvent DrugResistant Cancer by Acting on Mitochondrial Signaling Pathways. Biomaterials 34, 3366-3380. doi:10.1016/j.biomaterials.2013.01.055

Li, W., Zhang, H., Assaraf, Y. G., Zhao, K., Xu, X., Xie, J., et al. (2016). Overcoming Abc Transporter-Mediated Multidrug Resistance: Molecular Mechanisms and Novel Therapeutic Drug Strategies. Drug Resist. Updates 27, 14-29. doi:10.1016/j.drup.2016.05.001

Li, Y. C., Fung, K. P., Kwok, T. T., Lee, C. Y., Suen, Y. K., and Kong, S. K. (2002). Mitochondrial Targeting Drug Lonidamine Triggered Apoptosis in Doxorubicin-Resistant Hepg2 Cells. Life Sci. 71, 2729-2740. doi:10.1016/ s0024-3205(02)02103-3

Liu, Y., Zhang, X., Zhou, M., Nan, X., Chen, X., and Zhang, X. (2017). Mitochondrial-targeting Lonidamine-Doxorubicin Nanoparticles for Synergistic Chemotherapy to Conquer Drug Resistance. ACS Appl. Mater. Inter. 9, 43498-43507. doi:10.1021/acsami.7b14577

Liu, Z., Zhong, Y., Zhou, X., Huang, X., Zhou, J., Huang, D., et al. (2021). Inherently Nitric Oxide Containing Polymersomes Remotely Regulated by Nir for Improving Multi-Modal Therapy on Drug Resistant Cancer. Biomaterials 277, 121118. doi:10.1016/j.biomaterials.2021.121118

Majumder, J., Taratula, O., and Minko, T. (2019). Nanocarrier-based Systems for Targeted and Site Specific Therapeutic Delivery. Adv. Drug Deliv. Rev. 144, 57-77. doi:10.1016/j.addr.2019.07.010

Nath, K., Guo, L., Nancolas, B., Nelson, D. S., Shestov, A. A., Lee, S.-C., et al. (2016). Mechanism of Antineoplastic Activity of Lonidamine. Biochim. Biophys. Acta (Bba) - Rev. Cancer 1866, 151-162. doi:10.1016/j.bbcan.2016.08.001

Nath, K., Nelson, D. S., Heitjan, D. F., Leeper, D. B., Zhou, R., and Glickson, J. D. (2015). Lonidamine Induces Intracellular Tumor Acidification and ATP Depletion in Breast, Prostate and Ovarian Cancer Xenografts and Potentiates Response to Doxorubicin. NMR Biomed. 28, 281-290. doi:10.1002/nbm.3240

Nath, K., Nelson, D. S., Ho, A. M., Lee, S.-C., Darpolor, M. M., Pickup, S., et al. (2013). 31P and1H MRS of DB-1 Melanoma Xenografts: Lonidamine Selectively Decreases Tumor Intracellular $\mathrm{pH}$ and Energy Status and Sensitizes Tumors to Melphalan. NMR Biomed. 26, 98-105. doi:10.1002/nbm.2824

Peng, Y., Lu, J., Li, R., Zhao, Y., Hai, L., Guo, L., et al. (2021). Glucose and Triphenylphosphonium Co-modified Redox-Sensitive Liposomes to Synergistically Treat Glioma with Doxorubicin and Lonidamine. ACS Appl. Mater. Inter. 13, 26682-26693. doi:10.1021/acsami.1c02404

Price, G. S., Page, R. L., Riviere, J. E., Cline, J. M., and Thrall, D. E. (1996). Pharmacokinetics and Toxicity of Oral and Intravenous Lonidamine in Dogs. Cancer Chemother. Pharmacol. 38, 129-135. doi:10.1007/s002800050460

$\mathrm{Pu}$, X., Zhao, L., Li, J., Song, R., Wang, Y., Yu, K., et al. (2019). A Polymeric Micelle with an Endosomal Ph-Sensitivity for Intracellular Delivery and Enhanced Antitumor Efficacy of Hydroxycamptothecin. Acta Biomater. 88, 357-369. doi:10.1016/j.actbio.2019.02.039

Qiao, H., Chen, X., Chen, E., Zhang, J., Huang, D., Yang, D., et al. (2019). Folated Ph-Degradable Nanogels for the Simultaneous Delivery of Docetaxel and an Ido1-inhibitor in Enhancing Cancer Chemo-Immunotherapy. Biomater. Sci. 7, 2749-2758. doi:10.1039/c9bm00324j 
Qiao, Y., Wei, Z., Qin, T., Song, R., Yu, Z., Yuan, Q., et al. (2021). Combined Nanosuspensions from Two Natural Active Ingredients for Cancer Therapy with Reduced Side Effects. Chin. Chem. Lett. 32, 2877-2881. doi:10.1016/ j.cclet.2021.03.049

Rebucci, M., and Michiels, C. (2013). Molecular Aspects of Cancer Cell Resistance to Chemotherapy. Biochem. Pharmacol. 85, 1219-1226. doi:10.1016/ j.bcp.2013.02.017

Shahriari, M., Zahiri, M., Abnous, K., Taghdisi, S. M., Ramezani, M., and Alibolandi, M. (2019). Enzyme Responsive Drug Delivery Systems in Cancer Treatment. J. Controlled Release 308, 172-189. doi:10.1016/j.jconrel.2019.07.004

Tu, Z., Qiao, H., Yan, Y., Guday, G., Chen, W., Adeli, M., et al. (2018). Directed Graphene-Based Nanoplatforms for Hyperthermia: Overcoming Multiple Drug Resistance. Angew. Chem. Int. Ed. 57, 11198-11202. doi:10.1002/ anie.201804291

Yang, X., Hu, C., Tong, F., Liu, R., Zhou, Y., Qin, L., et al. (2019). Tumor Microenvironment-Responsive Dual Drug Dimer-Loaded PEGylated Bilirubin Nanoparticles for Improved Drug Delivery and Enhanced Immune-Chemotherapy of Breast Cancer. Adv. Funct. Mater. 29, 1901896. doi:10.1002/adfm.201901896

Zhang, B.-F., Xing, L., Cui, P.-F., Wang, F.-Z., Xie, R.-L., Zhang, J.-L., et al. (2015). Mitochondria Apoptosis Pathway Synergistically Activated by Hierarchical Targeted Nanoparticles Co-delivering Sirna and Lonidamine. Biomaterials 61, 178-189. doi:10.1016/j.biomaterials.2015.05.027

Zhong, Y., Zhang, J., Zhang, J., Hou, Y., Chen, E., Huang, D., et al. (2021). Tumor Microenvironment-Activatable Nanoenzymes for Mechanical Remodeling of Extracellular Matrix and Enhanced Tumor Chemotherapy. Adv. Funct. Mater. 31, 2007544. doi:10.1002/adfm.202007544
Zong, L., Wang, H., Hou, X., Fu, L., Wang, P., Xu, H., et al. (2021). A Novel GshTriggered Polymeric Nanomicelles for Reversing $\mathrm{Mdr}$ and Enhancing Antitumor Efficiency of Hydroxycamptothecin. Int. J. Pharmaceutics 600, 120528. doi:10.1016/j.ijpharm.2021.120528

Zong, L., Wang, Y., Qiao, P., Yu, K., Hou, X., Wang, P., et al. (2020). Reductionsensitive Poly(ethylene Glycol)-Polypeptide Conjugate Micelles for Highly Efficient Intracellular Delivery and Enhanced Antitumor Efficacy of Hydroxycamptothecin. Nanotechnology 31, 165102. doi:10.1088/1361-6528/ ab6749

Conflict of Interest: The authors declare that the research was conducted in the absence of any commercial or financial relationships that could be construed as a potential conflict of interest.

Publisher's Note: All claims expressed in this article are solely those of the authors and do not necessarily represent those of their affiliated organizations, or those of the publisher, the editors, and the reviewers. Any product that may be evaluated in this article, or claim that may be made by its manufacturer, is not guaranteed or endorsed by the publisher.

Copyright (c) 2021 Chen, Wang, Zhang, Zhou, Niu, Liu, Zhong, Huang and Chen. This is an open-access article distributed under the terms of the Creative Commons Attribution License (CC BY). The use, distribution or reproduction in other forums is permitted, provided the original author(s) and the copyright owner(s) are credited and that the original publication in this journal is cited, in accordance with accepted academic practice. No use, distribution or reproduction is permitted which does not comply with these terms. 